\title{
Multiple Fault Detection and Classification In Power System
}

\author{
1Dr. Afaneen A. Alkhazraji and ${ }^{2}$ Rana A.Abttan \\ ${ }^{1}$ Asst. Prof., University of Technology University of Technology, Electrical Eng. Department, Iraq. \\ ${ }^{2}$ Asst. Lecturer, Baghdad College for Economic Sciences University, Iraq.
}

Correspondence Author: Rana A. Abttan, Baghdad College for Economic Sciences University, Baghdad, Iraq. E-mail:Rana_iraq_9999@yahoo.com

Received date: 11 February 2018, Accepted date: 15 May 2018, Online date: 29 May 2018

Copyright: (C) 2018 Dr. Afaneen A. Alkhazraji and Rana A. Abttan. This is an open-access article distributed under the terms of the Creative Commons Attribution License, which permits unrestricted use, distribution, and reproduction in any medium, provided the original author and source are credited.

\begin{abstract}
Multiple Fault is a central component of more Abnormal Event Management in electric power systems. The main objective of multiple fault detection and classification satisfy high reliability degree to ensure continuity of supply of electric power as well as to reduce the time of separation of lines, reduce losses and consumer complaints. Detection and isolation of multiple faults in power systems is very important not only for service continuity but also for quality of power delivered. As an intermediate stage before fault location, fault classification is a crucial task in any reliable protective relay system This paper presents an approach for detection and classification for multiple fault in the electrical power system. Voltage and current measurement method is adapted in this work. Bus bar fault currents and voltages for different types of fault were detected and classified. All programs were written in MATLAB environment. The programs were test on IEEE- 11 bus bar network. The results clarified that the voltage and current measurement method is very effective for multiple fault detection and classification.
\end{abstract}

Key words: Multiple fault ; multiple fault analysis; multiple fault detection ; multiple fault type classification.

\section{INTRODUCTION}

An electrical power system consist of generation system, transmission system, sub transmission system, and distribution system .in general ,the generation and transmission systems are referred to as bulk power supply and the sub transmission and distribution systems are considered as the final means to transfer electric power to the ultimate customer. In power systems, high voltage transmission and distribution lines are vital links that achieve the essential continuity of service from generating plants to the end users. Rapid restoration of service could reduce customer complaints, outage time, and crew repair expense. all of these factors are increasingly important to researchers and utilities facing challenges in competitive electrical power. From views of economics and quality of power feeding, the importance of fault detection/location in power system is increasing.

When a fault occurs in power system, it is very important to detect it, determine its type, and find its location in order to make necessary repairs and to restore power as soon as possible. Detection and classification of faults help the system to satisfy high reliability degree. Fault detection enables faults to be sensed on bus bar by monitoring the voltage and current value. The four types of fault are signal line to earth faults, line to line faults, line to line to ground faults and three phase faults [1].

Multiple fault of particular interest is frequently occurring in a power system which is mixed of all type faults. Multiple fault may consist of two different types of fault at the same location or at different locations.

Several investigations have examined the fault detection and classification in power transmission line. Charles A. Gross [2] explain how conventional method can be used to calculation multiple fault for unsymmetrical at different locations. D. M. Gilbert et al. [3]an adaptive statistical estimator for the basis of detection and classification of power system faults. A. A. Girgis et al. [4] presented a Hybrid-Expert system for fault detection, classification, and fault selection location algorithms. Kezunovic et al. [5] implemented a study about the detection and classification of faults using ANNs. Saha et al. [6] designed a scheme of the fault diagnosis based on a suitable model working at real time. This model was to detect the fault at unsymmetrical case for two parts of the power system, transmission and distribution parts. Samantaray [7] proposed the systematic fuzzy rule as a new approach used for the classification method for the faults issues that occur on the transmission system. Recioui et al. [8] proposed the K-Nearest Neighbors for detection, classification and location of power system faults. S. Saeid Taheri, and Hosein Askarian-Abyaneh [9] proposed coordinate Power Swing Blocking relays (PSBs) for worst out of step condition that may occurs in a system. We could simulate that condition using software that programmed for simulating of two simultaneous fault conditions, for the first time.

In this work a conventional method is adopted for detect, classify, and locate symmetrical and unsymmetrical of multiple faults in power system.

Multiple Fault Analysis:

A three-phase power system may be modeled as three single-phase symmetrical component or "sequence" electrical networks, described by described by

$\mathrm{v}_{0}=-\left[\mathrm{Z}_{0}\right] \mathrm{I}_{0}$

$\mathrm{v}_{1}=\mathrm{E}_{1}-\left[\mathrm{Z}_{1}\right] \mathrm{I}_{1}$

$1 \mathrm{~A}$

$\mathrm{v}_{2}=-\left[\mathrm{Z}_{2}\right] \mathrm{I}_{2}$

Where $\mathrm{v}_{0}, \mathrm{v}_{1}$, and $\mathrm{v}_{2}$ are ( $\left.\mathrm{n} \boldsymbol{x} 1\right)$ sequence phasor voltage vectors 
Citation: Dr. Afaneen A. Alkhazraji and Rana A.Abttan, 2018. Multiple Fault Detection And Classification In Power System. Advances in Natural and Applied Sciences., 12(5): 7-13.

$\mathrm{I}_{0}, \mathrm{I}_{1}$, and $\mathrm{I}_{2}$ are (n $\boldsymbol{x} 1$ ) sequence phasor current vectors

$E_{1}$ isPre-fault positive sequence voltage vector (n $x 1$ )

$\left[Z_{0}\right],\left[Z_{1}\right]$ and $\left[Z_{2}\right]$ are (n $x \mathrm{n}$ ) sequence impedance matrices (commonly called "Z bus").

$\mathrm{n}=$ number of busses (nodes) in the system.

all values in per-unit.

Defining faults is straight forward. For purposes of discussion, consider a single line to ground fault at bus i and a phase-to-phase fault at bus j. From equation (1). $\mathrm{Z}_{012}$ can be formulated as [2]:

$\left[\mathrm{Z}_{012}\right]=\left[\begin{array}{cccccc}\mathrm{z}_{\mathrm{ii}}^{0} & 0 & 0 & \mathrm{z}_{\mathrm{jj}}^{0} & 0 & 0 \\ 0 & \mathrm{z}_{\mathrm{ii}}^{1} & 0 & 0 & \mathrm{z}_{\mathrm{jj}}^{1} & 0 \\ 0 & 0 & \mathrm{z}_{\mathrm{ii}}^{2} & 0 & 0 & \mathrm{z}_{\mathrm{jj}}^{2} \\ \mathrm{z}_{\mathrm{ji}}^{0} & 0 & 0 & \mathrm{z}_{\mathrm{jj}}^{0} & 0 & 0 \\ 0 & \mathrm{z}_{\mathrm{ji}}^{1} & 0 & 0 & \mathrm{z}_{\mathrm{jj}}^{1} & 0 \\ 0 & 0 & \mathrm{z}_{\mathrm{ji}}^{2} & 0 & 0 & \mathrm{z}_{\mathrm{jj}}^{2}\end{array}\right]$

Where $\left[Z_{012}\right]$ is sequence impedance matrix and formulated from entries from $\left[Z_{0}\right],\left[Z_{1}\right]$ and $\left[Z_{2}\right]$.then:

$\left[\mathrm{Z}_{\mathrm{abc}}\right]=\left[\mathrm{T}^{-1}\left[\mathrm{Z}_{012}\right][\mathrm{T}]\right.$

$[\mathrm{T}]=\left[\begin{array}{ll}{[\mathrm{T}]} & {[0]} \\ {[0]} & {[\mathrm{T}]}\end{array}\right]$

$[\mathrm{T}]=\left[\begin{array}{ccc}1 & 1 & 1 \\ 1 & \mathrm{a}^{2} & \mathrm{a} \\ 1 & \mathrm{a} & \mathrm{a}^{2}\end{array}\right]$

Where $\left[\mathrm{Z}_{\mathrm{abc}}\right]$ is the phase impedance matrix and $\mathrm{T}$ is the symmetrical components transformation matrix?

Likewise:

$\left[\mathrm{E}_{\mathrm{abc}}\right]=[\mathrm{T}]\left[\begin{array}{llllll}0 & 1 & 0 & 0 & 1 & 0\end{array}\right]^{\mathrm{t}}$

$=\left[\begin{array}{llllll}1 & a^{2} & a & 1 & a^{2} & a\end{array}\right]^{t}$

$\left[V_{a b c}\right]=\left[V_{i}^{a} V_{i}^{b} V_{i}^{c} V_{j}^{a} V_{j}^{b} V_{j}^{c}\right]^{t}$

$\left[I_{a b c}\right]=\left[I_{i}^{a} I_{i}^{b} I_{i}^{c} I_{j}^{a} I_{j}^{b} I_{j}^{c}\right]^{t}$

Where $\left[\mathrm{E}_{\mathrm{abc}}\right]$ is the phase internal voltages of synchronous machine, $\left[\mathrm{V}_{\mathrm{abc}}\right]$ is the phase terminal voltages vector and $\left[\mathrm{I}_{\mathrm{abc}}\right]$ is the phase current vector?

Finally

$\mathrm{E}_{\mathrm{abc}}-\mathrm{V}_{\mathrm{abc}}=\mathrm{U}_{\mathrm{abc}}=\left[\mathrm{Z}_{\mathrm{abc}}\right] \mathrm{I}_{\mathrm{abc}}$

Where $U_{a b c}$ is the phase voltage change between synchronous machine and bus-bar.

Mathematical model:

A.Fault Detection:

The abnormal conditions are detected by watching the phase impedances and/or phase-current amplitudes and/or phase-voltage amplitudes and/or zerosequence current amplitude. It is difference between pre fault measurement and fault measurement as shown:

If

$\mathrm{V}_{\text {bus }}(0)=\left[\begin{array}{c}\mathrm{V}_{1}(0) \\ \mathrm{V}_{\mathrm{k}}(0) \\ \mathrm{V}_{\mathrm{n}}(0)\end{array}\right]$

Where $V_{\text {bus }}(0)$ is the prefault voltages obtained from power flow solution

And

$\Delta \mathrm{V}_{\text {bus }}=\left[\begin{array}{c}\Delta \mathrm{V}_{1} \\ \Delta \mathrm{V}_{\mathrm{k}} \\ \Delta \mathrm{V}_{\mathrm{n}}\end{array}\right]$

Where $\Delta V_{\text {bus }}$ is the bus voltage changes caused by the fault. Then :

$\mathrm{V}_{\text {bus }}(\mathrm{f})=\mathrm{V}_{\text {bus }}(0)+\Delta \mathrm{V}_{\text {bus }}$

$\mathrm{V}_{\text {bus }}(\mathrm{f})$ is the voltages during the fault. Which lead to:

$\left[\begin{array}{c}0 \\ \vdots \\ \mathrm{I}_{\mathrm{k}}(\mathrm{f}) \\ \vdots \\ 0\end{array}\right]=\left[\begin{array}{cccc}\mathrm{y}_{11} & \cdots & \mathrm{y}_{1 \mathrm{k}} \cdots & \mathrm{y}_{1 \mathrm{n}} \\ \cdot & \cdot & \cdot & \cdot \\ \mathrm{y}_{\mathrm{k} 1} & \cdots & \mathrm{y}_{\mathrm{kk}} \cdots & \mathrm{y}_{\mathrm{kn}} \\ \cdot & \cdot & \cdot & \cdot \\ \mathrm{y}_{\mathrm{n} 1} & \cdots & \mathrm{y}_{\mathrm{nk}} \cdots & \mathrm{y}_{\mathrm{nn}}\end{array}\right]\left[\begin{array}{c}\Delta \mathrm{v}_{1} \\ \cdot \\ \Delta \mathrm{v}_{\mathrm{k}} \\ \cdot \\ \Delta \mathrm{v}_{\mathrm{n}}\end{array}\right]$

Where $\mathrm{I}_{\text {bus }}$ is the bus current entering the bus and $\mathrm{Y}_{\text {bus }}$ is the bus admittance matrix.

B. Fault Classification:

Fault classification technique used in electric power system is vital for secure operation of power systems and has to be accurate to facilitate quick repair of the system. Idea and purpose is to classify fault based on magnitude of current [10].

1- if one phase : SLG (Single Line to Ground)

ag: $\mathrm{Ia}=$ value, $\mathrm{Ib}=\mathrm{Ic}=0$

bg: $\mathrm{Ib}=$ value, $\mathrm{Ia}=\mathrm{Ic}=0$

cg: Ic=value, $\mathrm{Ia}=\mathrm{Ib}=0$

2- if two phase :

i. $\quad \mathrm{L}-\mathrm{L}$ (Line to Line), $\mathrm{I}_{\mathrm{a} 0}=0$

$\mathrm{ab}: \mathrm{Ia}=-\mathrm{Ib}=$ value $, \mathrm{Ic}=0, \mathrm{I}_{\mathrm{a} 0}=0$

$\mathrm{bc}: \mathrm{Ib}=-\mathrm{Ic}=$ value, $\mathrm{Ia}=0, \mathrm{I}_{\mathrm{a} 0}=0$

ca: $\mathrm{Ic}=-\mathrm{Ia}=$ value $, \mathrm{Ib}=0, \mathrm{I}_{\mathrm{a} 0}=0$

ii. DLG (Double Line to Ground), $\mathrm{I}_{\mathrm{a} 0}=$ value

$\mathrm{abg}: \mathrm{Ia}=-\mathrm{Ib}=$ value $, \mathrm{Ic}=0, \mathrm{I}_{\mathrm{a} 0}=$ value

bcg $: I b=-I c=$ value, $I a=0, I_{\mathrm{a} 0}=$ value

cag: $\mathrm{Ic}=-\mathrm{Ia}=$ value $, \mathrm{Ib}=0, \mathrm{I}_{\mathrm{a} 0}=$ value

ifthreephase: $3 \Phi: \mathrm{a}$ b c: $\mathrm{Ia}=\mathrm{Ib}=\mathrm{Ic}=$ value, $\mathrm{I}_{\mathrm{a} 0}=0, \mathrm{I}_{\mathrm{a} 2}=0$

The structure of the proposed diagram:

A program was written in MATLAB environment for the detection and classification and finds the location of multiple faults. The input data was calculated by a Newton-Raphson load flow program and considered as the initials values. Shown in "Fig. 1,"is described as follows: 
Citation: Dr. Afaneen A. Alkhazraji and Rana A.Abttan, 2018. Multiple Fault Detection And Classification In Power System. Advances in Natural and Applied Sciences., 12(5): 7-13.

Step1: Input data of power transmission system including all the line data, bus data and G\&T data.

Step2: Run the load flow program to calculate the initial values of currents and voltages

Step 3: Building Z-Bus programs which are needed

Step4: run multiple fault analysis programs and calculate current bus bar.

Step5: compare current value before and after fault.

Step6: fault detection and classification based on magnitude of current End

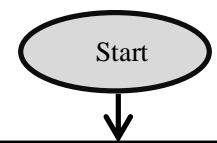

Read Bus Data, Line Data, G. \&T. Data, $\mathrm{n}=$ no. of buses, $\mathrm{m}=$ no. of phases

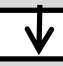

Run load flow (for calculate voltage for each bus bar for calculate current for earh huc har hefore fault)

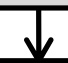

Formulate \& Store Z-Bus1, Z-Bus2 \& Z-Bus0

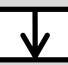

Store current for each bus bar before fault in matrix $\mathrm{Q}(\mathrm{n}, \mathrm{m})$

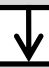

Run multiple fault analysis Program

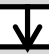

Calculate and store current for each bus bar in matrix W (n, m) after fault

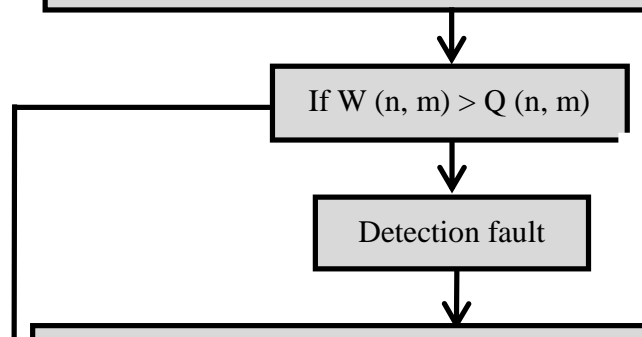

Fault classification according to number phases in matrix $\mathrm{W}$

1-if one phase: SLG (ag, bg, cg)

2-if two phase: $\mathrm{L}-\mathrm{L}, \mathrm{Ia} 0=0 \quad(\mathrm{ab}, \mathrm{bc}, \mathrm{ac})$

Ia0 =value $\quad(a b g, b c g, a c g)$, DLG

3-if 3phase: $3 \Phi$ (abcg)

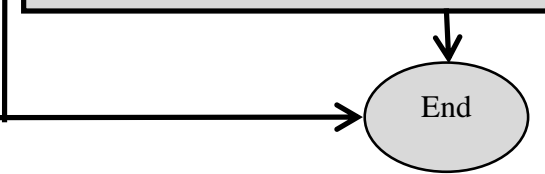

Fig. 1: Flow chart of conventional method

Case study:

To clarify the effectiveness of the proposed program, the program was applied on the IEEE 11- bus test system. The system consists of 11 bus and 14 transmission line. The single line diagram of this system and its data are explained in the appendix (A). Table-1 shows Power Flow Solution by Newton-Raphsonprogram.

Table 1: power flow solution by Newton-Raphson method

\begin{tabular}{|c|c|c|c|c|c|c|c|}
\hline \multirow{2}{*}{\begin{tabular}{|l} 
Bus \\
No. \\
\end{tabular}} & \multirow{2}{*}{\begin{tabular}{|l|} 
Voltage \\
Mag. \\
\end{tabular}} & \multirow{2}{*}{\begin{tabular}{|l|} 
Angle \\
Degree \\
\end{tabular}} & \multicolumn{2}{|c|}{-------Load------- } & \multicolumn{3}{|c|}{---Generation--- } \\
\hline & & & MW & Mvar & MW & Mvar & Mvar \\
\hline 1 & 1.04 & 0 & 0 & 0 & 240.01 & 211.387 & 0 \\
\hline 2 & 1.028 & -0.772 & 0 & 0 & 0 & 0 & 0 \\
\hline 3 & 1.004 & -2.26 & 150 & 120 & 0 & 0 & 0 \\
\hline 4 & 1.024 & -0.587 & 0 & 0 & 0 & 0 & 0 \\
\hline 5 & 1.02 & -1.413 & 120 & 60 & 0 & 0 & 0 \\
\hline 6 & 1 & -2.588 & 140 & 90 & 0 & 0 & 0 \\
\hline 7 & 1.021 & -0.425 & 0 & 0 & 0 & 0 & 0 \\
\hline 8 & 0.993 & -2.765 & 110 & 90 & 0 & 0 & 0 \\
\hline 9 & 0.99 & -3.174 & 80 & 50 & 0 & 0 & 0 \\
\hline 10 & 1.035 & 0.278 & 0 & 0 & 200 & 147.649 & 0 \\
\hline 11 & 1.03 & 0.447 & 0 & 0 & 160 & 92.906 & 0 \\
\hline Total & & & 600 & 410 & 600.01 & 451.943 & 0 \\
\hline
\end{tabular}


Citation: Dr. Afaneen A. Alkhazraji and Rana A.Abttan, 2018. Multiple Fault Detection And Classification In Power System. Advances in Natural and Applied Sciences., 12(5): 7-13

Two types of fault have been considered to verify the accuracy of the proposed program, those are:

-Unsymmetrical fault: Single line to ground fault at bus 3 and line to line fault at bus 9

Table 2,3 show comparisons of bus bar voltages (amplitude and phase) and transmission currents, respectively, before and after the occurrence of the unsymmetrical multiple faults on busses 3 and 9 .

Table 2: Comparison of voltages of an unsymmetrical multiple fault on bus 3 and 9

\begin{tabular}{|c|c|c|c|c|c|c|c|c|}
\hline \multirow{3}{*}{\begin{tabular}{|l|} 
bus \\
\\
no. \\
\end{tabular}} & \multicolumn{2}{|c|}{ before fault } & \multicolumn{6}{|c|}{ when fault at two bus bar 3,9 [after fault] } \\
\hline & & & \multicolumn{2}{|l|}{$\mathrm{a}$} & \multicolumn{2}{|l|}{$\mathrm{b}$} & \multicolumn{2}{|l|}{$\mathrm{c}$} \\
\hline & Voltage & Angle & Voltage & Angle & Voltage & Angle & Voltage & Angle \\
\hline & Mag. & Degree & Mag. & Degree & Mag. & Degree & Mag. & Degree \\
\hline 1 & 1.04 & 0 & 0.8295 & -0.1067 & 0.8021 & -120.7342 & 0.8084 & 121.2674 \\
\hline 2 & 1.028 & -0.772 & 0.7029 & 0.743 & 0.7476 & -123.2629 & 0.7542 & 126.3063 \\
\hline 3 & 1.004 & -2.26 & 0.0484 & 146.699 & 0.826 & -140.9367 & 0.8254 & 147.7862 \\
\hline 4 & 1.024 & -0.587 & 0.6253 & -1.3788 & 0.6701 & -137.1911 & 0.7029 & 142.6522 \\
\hline 5 & 1.02 & -1.413 & 0.722 & 1.2984 & 0.7173 & -124.2178 & 0.7339 & 129.7768 \\
\hline 6 & 1 & -2.588 & 0.4271 & 2.7774 & 0.6266 & -138.4035 & 0.6822 & 149.4184 \\
\hline 7 & 1.021 & -0.425 & 0.8027 & -0.8948 & 0.6661 & -131.4554 & 0.7016 & 136.0831 \\
\hline 8 & 0.993 & -2.765 & 0.5683 & -1.0752 & 0.3988 & -173.7477 & 0.6062 & -176.8336 \\
\hline 9 & 0.99 & -3.174 & 0.448 & -11.331 & 0.997 & 109.0466 & 1.2891 & -124.7212 \\
\hline 10 & 1.035 & 0.278 & 0.7681 & -1.6738 & 0.8028 & -130.5501 & 0.8117 & 132.1888 \\
\hline 11 & 1.03 & 0.447 & 0.9083 & -1.0356 & 0.7491 & -126.2289 & 0.7758 & 126.8671 \\
\hline
\end{tabular}

Table 3: Comparison of currents of a symmetrical multiple faults on bus 3 and 9

\begin{tabular}{|c|c|c|c|c|c|c|c|c|c|c|c|c|c|}
\hline \multirow{3}{*}{\begin{tabular}{|l} 
Bus \\
No. \\
\end{tabular}} & \multirow{3}{*}{\begin{tabular}{|l|} 
Bus \\
No. \\
\end{tabular}} & \multicolumn{6}{|c|}{ Line current before fault } & \multicolumn{6}{|c|}{ Line current after fault (on bus 3,9 ) } \\
\hline & & \multicolumn{2}{|l|}{$\mathrm{a}$} & \multicolumn{2}{|l|}{$\mathrm{b}$} & \multicolumn{2}{|l|}{$\mathrm{c}$} & \multicolumn{2}{|l|}{$\mathrm{a}$} & \multicolumn{2}{|l|}{$\mathrm{b}$} & \multicolumn{2}{|l|}{$\mathrm{c}$} \\
\hline & & Current & Angle & Current & Angle & Current & Angle & Current & Angle & Current & Angle & Current & Angle \\
\hline & & Mag. & Degree & Mag. & Degree & Mag. & Degree & Mag. & Degree & Mag. & Degree & Mag. & Degree \\
\hline 1 & 2 & 0.3076 & -138.6202 & 0.3076 & 101.3798 & 0.3076 & -18.6202 & 1.2757 & -100.0697 & 1.4049 & 142.6643 & 1.3996 & 16.7765 \\
\hline 2 & 3 & 0.1192 & -136.0462 & 0.1192 & 103.9538 & 0.1192 & -16.0462 & 1.7983 & -92.6667 & 0.3846 & -166.4964 & 0.5568 & -16.4771 \\
\hline 2 & 5 & 0.0947 & -142.7078 & 0.0947 & 97.2922 & 0.0947 & -22.7078 & 0.1346 & 113.3812 & 0.2221 & 169.2544 & 0.3266 & -28.5106 \\
\hline 2 & 6 & 0.094 & -137.7683 & 0.094 & 102.2317 & 0.094 & -17.7683 & 0.4396 & -95.2939 & 0.4396 & -95.2939 & 0.4817 & 174.5208 \\
\hline 3 & 4 & 0.0892 & 35.3779 & 0.0892 & -84.6221 & 0.0892 & 155.3779 & 1.2706 & 85.1148 & 0.1605 & -171.5883 & 0.062 & -51.9947 \\
\hline 3 & 6 & 0.0166 & -147.0635 & 0.0166 & 92.9365 & 0.0166 & -27.0635 & 0.855 & 88.8527 & 0.2851 & 155.3072 & 0.2373 & -8.5492 \\
\hline 4 & 6 & 0.0706 & -145.0056 & 0.0706 & 94.9944 & 0.0706 & -25.0056 & 0.2918 & -101.9638 & 0.107 & 128.498 & 0.1313 & -0.9949 \\
\hline 4 & 9 & 0.0805 & -141.9345 & 0.0805 & 98.0655 & 0.0805 & -21.9345 & 0.244 & -71.7686 & 2.0428 & 172.3677 & 2.1061 & -5.912 \\
\hline 4 & 10 & 0.2402 & 36.1653 & 0.2402 & \begin{tabular}{|l|}
-83.8347 \\
\end{tabular} & 0.2402 & 156.1653 & 1.7857 & 87.0348 & 1.9692 & -11.082 & 2.1946 & 175.5484 \\
\hline 5 & 7 & 0.0411 & 6.0835 & 0.0411 & -113.9165 & 0.0411 & 126.0835 & 0.1957 & 71.2846 & 0.2403 & -157.9793 & 0.1954 & -26.147 \\
\hline 6 & 8 & 0.0175 & -108.8682 & 0.0175 & 131.1318 & 0.0175 & 11.1318 & 0.2336 & 77.1858 & 0.7941 & 173.9887 & 0.7864 & -7.6547 \\
\hline 7 & 8 & 0.1429 & -143.6815 & 0.1429 & 96.3185 & 0.1429 & -23.6815 & 0.533 & -93.7755 & 1.3583 & 168.7308 & 1.4639 & -5.7807 \\
\hline 7 & 11 & 0.1796 & 29.7025 & 0.1796 & -90.2975 & 0.1796 & 149.7025 & 0.589 & 77.9975 & 1.2378 & -23.6482 & 1.2589 & -176.3725 \\
\hline 8 & 9 & 0.0154 & -160.0585 & 0.0154 & 79.9415 & 0.0154 & -40.0585 & 0.2418 & -59.4145 & 2.1022 & 174.3065 & 2.1098 & -5.6596 \\
\hline
\end{tabular}

- Symmetrical fault: 3 - phase fault on bus 5 and 3- phase fault at bus 8 :

Table 4, 5 show comparisons of bus bar voltages (amplitude and phase) and transmission currents before and after the symmetrical multiple faults occurrence on bus 5 and 8 , respectively.

Table 4: Comparison of voltages for multiple faults on bus 5 and 8

\begin{tabular}{|c|c|c|c|c|c|c|c|c|}
\hline \multirow{3}{*}{\begin{tabular}{|l|} 
bus \\
no. \\
\end{tabular}} & \multicolumn{2}{|c|}{ before fault } & \multicolumn{6}{|c|}{ when fault at two bus bar 5,8 [after fault] } \\
\hline & & & \multicolumn{2}{|l|}{$\mathrm{a}$} & \multicolumn{2}{|l|}{$\mathrm{b}$} & \multicolumn{2}{|l|}{$\mathrm{c}$} \\
\hline & Voltage & Angle & Voltage & Angle & Voltage & Angle & Voltage & Angle \\
\hline & Mag. & Degree & Mag. & Degree & Mag. & Degree & Mag. & Degree \\
\hline 1 & 1.04 & 0 & 0.5669 & 0 & 0.5669 & -120 & 0.5669 & 120 \\
\hline 2 & 1.028 & -0.772 & 0.413 & 1.9211 & 0.413 & -118.0789 & 0.413 & 121.921 \\
\hline 3 & 1.004 & -2.26 & 0.4636 & 4.8982 & 0.4636 & -115.1018 & 0.4636 & 124.898 \\
\hline 4 & 1.024 & -0.587 & 0.6756 & 0.8893 & 0.6756 & -119.1107 & 0.6756 & 120.889 \\
\hline 5 & 1.02 & -1.413 & 0.0316 & 52.647 & 0.0316 & -67.353 & 0.0316 & 172.647 \\
\hline 6 & 1 & -2.588 & 0.3668 & 7.0738 & 0.3668 & -112.926 & 0.3668 & 127.074 \\
\hline 7 & 1.021 & -0.425 & 0.3765 & 1.1525 & 0.3765 & -118.8475 & 0.3765 & 121.153 \\
\hline 8 & 0.993 & -2.765 & 0.0486 & 100.14 & 0.0486 & -19.8617 & 0.0486 & -139.86 \\
\hline 9 & 0.99 & -3.174 & 0.2599 & 12.179 & 0.2599 & -107.8211 & 0.2599 & 132.179 \\
\hline 10 & 1.035 & 0.278 & 0.808 & -0.357 & 0.808 & -120.3565 & 0.808 & 119.644 \\
\hline 11 & 1.03 & 0.447 & 0.5696 & -0.808 & 0.5696 & -120.8079 & 0.5696 & 119.192 \\
\hline
\end{tabular}


11

Citation: Dr. Afaneen A. Alkhazraji and Rana A.Abttan, 2018. Multiple Fault Detection And Classification In Power System. Advances in Natural and Applied Sciences., 12(5): 7-13.

Table 5: Comparison of currents of a symmetrical multiple fault on bus 5and 8

\begin{tabular}{|c|c|c|c|c|c|c|c|c|c|c|c|c|c|}
\hline \multirow{3}{*}{$\begin{array}{c}\text { Bus } \\
\text { No. }\end{array}$} & \multirow{3}{*}{$\begin{array}{c}\text { Bus } \\
\text { No. }\end{array}$} & \multicolumn{6}{|c|}{ Line current before fault } & \multicolumn{6}{|c|}{ Line current after fault(on bus 5,8 ) } \\
\hline & & \multicolumn{2}{|r|}{$\mathrm{a}$} & \multicolumn{2}{|c|}{$\mathrm{b}$} & \multicolumn{2}{|c|}{$\mathrm{c}$} & \multicolumn{2}{|c|}{$\mathrm{a}$} & \multicolumn{2}{|c|}{$\mathrm{b}$} & \multicolumn{2}{|c|}{$\mathrm{c}$} \\
\hline & & $\begin{array}{c}\text { Current } \\
\text { Mag. }\end{array}$ & $\begin{array}{l}\text { Angle } \\
\text { Degree }\end{array}$ & $\begin{array}{c}\text { Current } \\
\text { Mag. }\end{array}$ & $\begin{array}{l}\text { Angle } \\
\text { Degree }\end{array}$ & $\begin{array}{c}\text { Current } \\
\text { Mag. }\end{array}$ & $\begin{array}{l}\text { Angle } \\
\text { Degree }\end{array}$ & $\begin{array}{c}\text { Current } \\
\text { Mag. }\end{array}$ & $\begin{array}{l}\text { Angle } \\
\text { Degree }\end{array}$ & $\begin{array}{l}\text { Current } \\
\text { Mag. }\end{array}$ & $\begin{array}{c}\text { Angle } \\
\text { Degree }\end{array}$ & $\begin{array}{c}\text { Current } \\
\text { Mag. }\end{array}$ & $\begin{array}{c}\text { Angle } \\
\text { Degree }\end{array}$ \\
\hline 1 & 2 & 0.3076 & -138.6202 & 0.3076 & 101.3798 & 0.3076 & -18.6202 & 2.5790 & -95.1337 & 2.5790 & 144.8663 & 2.5790 & 24.8663 \\
\hline 2 & 3 & 0.1192 & -136.0462 & 0.1192 & 103.9538 & 0.1192 & -16.0462 & 0.1849 & 117.6464 & 0.1849 & -2.3536 & 0.1849 & -122.3536 \\
\hline 2 & 5 & 0.0947 & -142.7078 & 0.0947 & 97.2922 & 0.0947 & -22.7078 & 2.6252 & -91.6447 & 2.6252 & 148.3553 & 2.6252 & 28.3553 \\
\hline 2 & 6 & 0.0940 & -137.7683 & 0.0940 & 102.2317 & 0.0940 & -17.7683 & 0.1288 & -122.7077 & 0.1288 & 117.2923 & 0.1288 & -2.7077 \\
\hline 3 & 4 & 0.0892 & 35.3779 & 0.0892 & -84.6221 & 0.0892 & 155.3779 & 0.5389 & 82.2410 & 0.5389 & -37.7590 & 0.5389 & -157.7590 \\
\hline 3 & 6 & 0.0166 & -147.0635 & 0.0166 & 92.9365 & 0.0166 & -27.0635 & 0.2452 & -93.2635 & 0.2452 & 146.7365 & 0.2452 & 26.7365 \\
\hline 4 & 6 & 0.0706 & -145.0056 & 0.0706 & 94.9944 & 0.0706 & -25.0056 & 0.5224 & -96.3535 & 0.5224 & 143.6465 & 0.5224 & 23.6465 \\
\hline 4 & 9 & 0.0805 & -141.9345 & 0.0805 & 98.0655 & 0.0805 & -21.9345 & 0.6054 & -96.0075 & 0.6054 & 143.9925 & 0.6054 & 23.9925 \\
\hline 4 & 10 & 0.2402 & 36.1653 & 0.2402 & -83.8347 & 0.2402 & 156.1653 & 1.6666 & 83.3177 & 1.6666 & -36.6823 & 1.6666 & -156.6823 \\
\hline 5 & 7 & 0.0411 & 6.0835 & 0.0411 & -113.9165 & 0.0411 & 126.0835 & 0.8318 & 87.1836 & 0.8318 & -32.8164 & 0.8318 & -152.8164 \\
\hline 6 & 8 & 0.0175 & -108.8682 & 0.0175 & 131.1318 & 0.0175 & 11.1318 & 0.7762 & -90.4166 & 0.7762 & 149.5834 & 0.7762 & 29.5834 \\
\hline 7 & 8 & 0.1429 & -143.6815 & 0.1429 & 96.3185 & 0.1429 & -23.6815 & 1.1060 & -95.9769 & 1.1060 & 144.0231 & 1.1060 & 24.0231 \\
\hline 7 & 11 & 0.1796 & 29.7025 & 0.1796 & -90.2975 & 0.1796 & 149.7025 & 1.9371 & 85.3797 & 1.9371 & -34.6203 & 1.9371 & -154.6203 \\
\hline 8 & 9 & 0.0154 & -160.0585 & 0.0154 & 79.9415 & 0.0154 & -40.0585 & 0.5474 & 91.5172 & 0.5474 & -28.4828 & 0.5474 & -148.4828 \\
\hline
\end{tabular}

From observing the tables, it is very clear that there is a decrease in the voltage values of the bus bars near the fault location, because of voltage drop increment. The bus voltage on which the fault occurred is zero.

Also, whenever the fault location is away from the power supply, the Current fault value was relatively low compared with current fault value when fault occurred site near power supply.

The results given by a matlab simulation from the fault detection and classification.

Fig . 2 show fault detection on bus bar 3,9 and classification fault A-G on bus 3, B-C on bus 9 and fig . 3 show fault detection on bus bar 5,8 and classification fault A-B-C-G on bus 5, A-B-C-G on bus 8 .

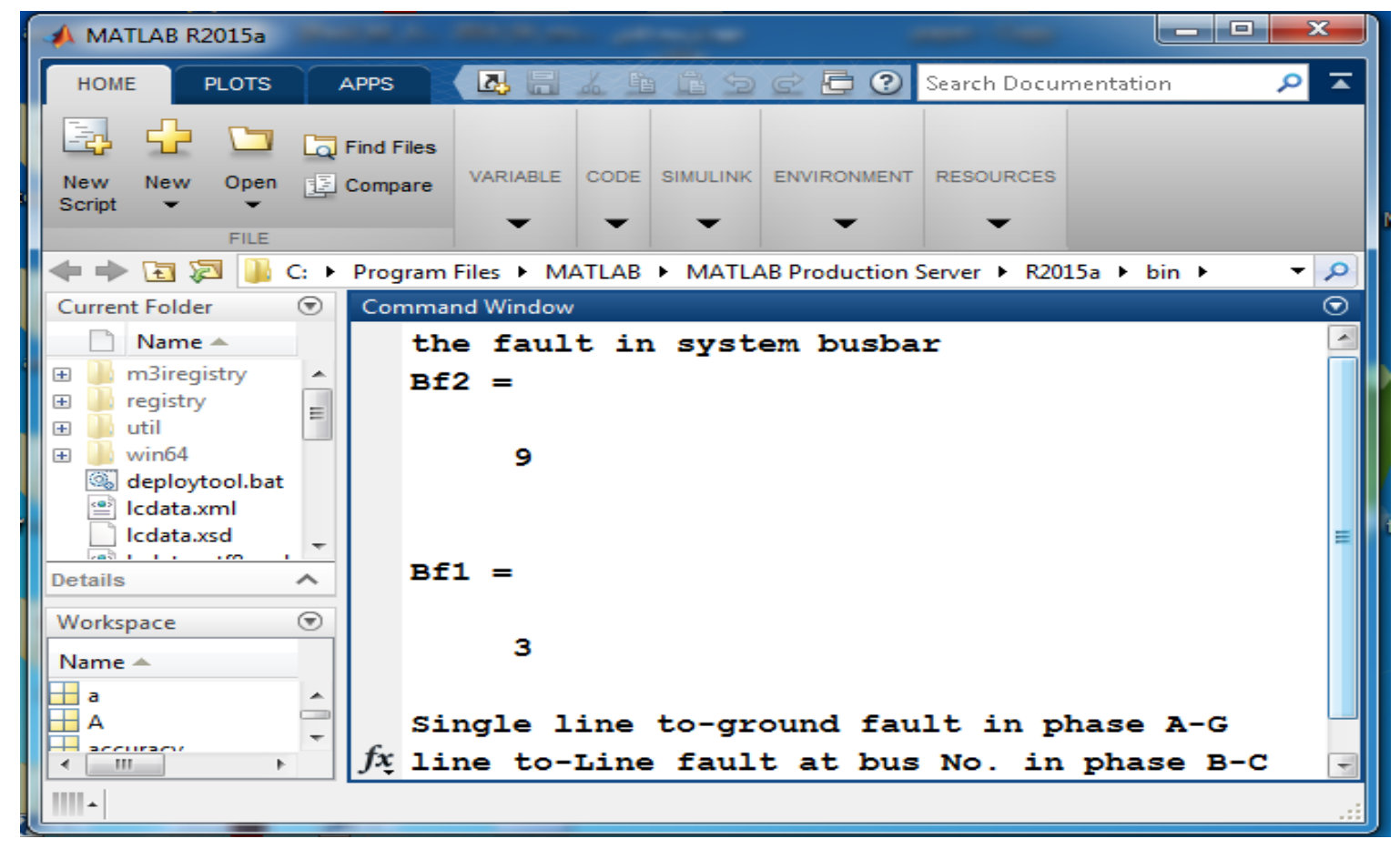

Fig. 2: results given by MATLAB program 


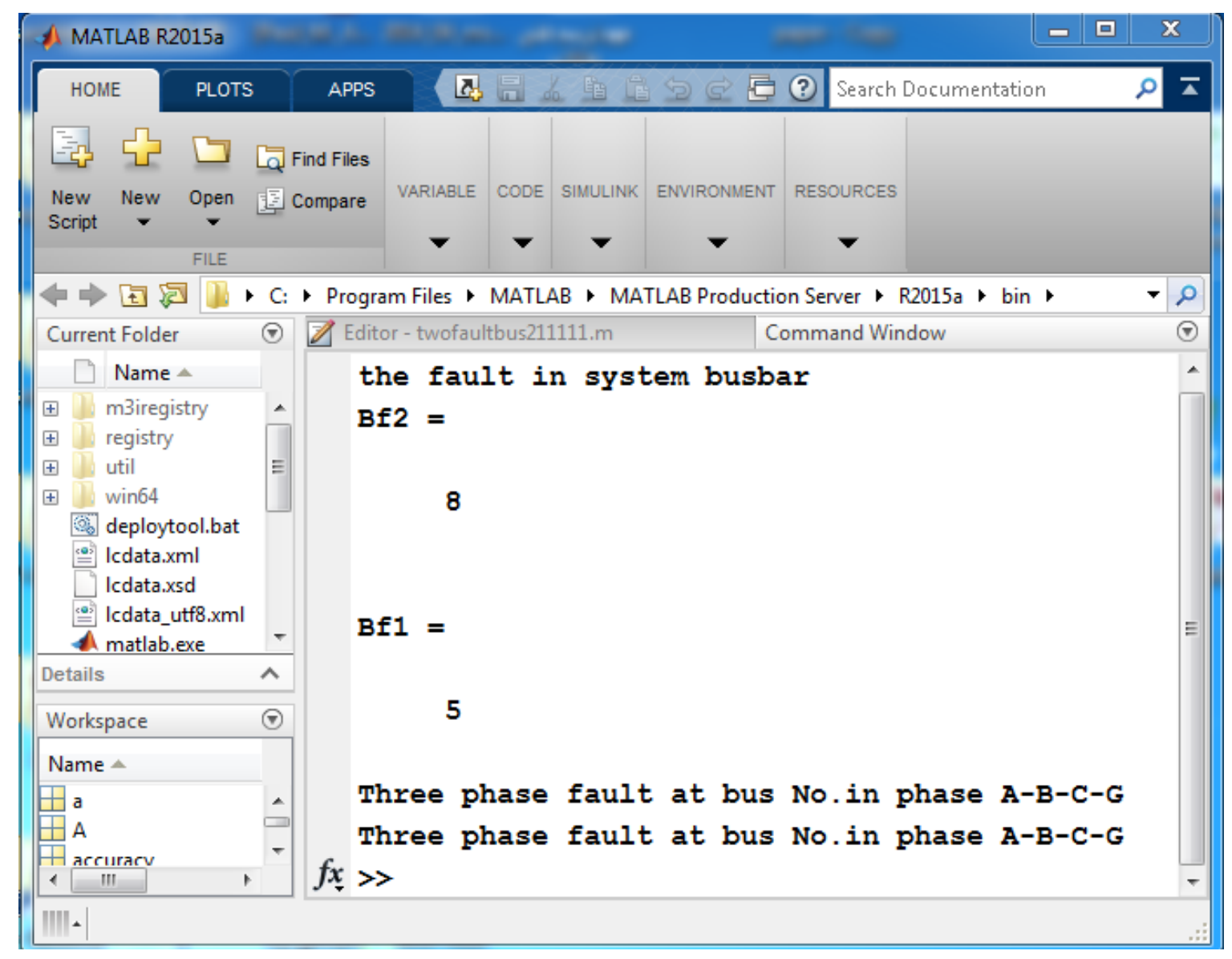

Fig. 3: results given by a MATLAB program

Conclusions:

The problem of multiple fault detection and classification in power system great importance for the economic operation of the power systems in nowadays competitive electricity markets. It provides a quick repair of the system which improves the system availability, and performance, and restoration time reduction and effort of maintenance. Hence, it increases the system reliability. In this work, a conventional method using current and voltage values comparisons has been presented. The obtained results show the effectiveness and the accuracy of this method for multiple fault detection and classification.

\section{REFERENCES}

[1] Thompson Adu, Senior Member, 2002. An Accurate Fault Classification Technique for Power System Monitoring Devices, IEEE Transaction on power system delivery, 17: 3 .

[2] Gilbert, D.M. and I.F. Morrison, 1997. A statistical method for the detection of power system faults, Elect. Power Energy Syst., 19: 269-275.

[3] Charles, A. Gross, 1990. Fault Calculations in Power Systems Subject to Multiple Faults, IEEE Trans. on Power Delivery, pp: 598-601.

[4] Girgis, A.A., M.B. Johnes, 1989. A Hybrid Expert System for Faulted Section Identification, Fault Type Classification and Selection of Fault Location Algorithms, IEEE Transaction on power system delivery, $4: 2$.

[5] Kezunovic, M., I. Rikalo, 1996. Detect and Classify Faults Using Neural Networks, IEEE computer applications in power, 7: 2.

[6] Saha, S., M. Aldeen and C.P. Tan, 2012. Unsymmetrical fault diagnosis in transmission/distribution networks, International Journal of Electrical Power \& Energy Systems, Volume 45, Issue 1, February, pp: 252-263.

[7] Samantaray, S.R., 2012. A systematic fuzzy rule based approach for fault classification in transmission lines, Applied Soft Computing, Volume 13, Issue 2, February, pp: 928-938.

[8] Recioui, A., B. Benseghier and H. Khalfallah, 2015. Power System Fault Detection, Classification and Location Using the K- Nearest Neighbors, IEEE International Conference on Electrical Engineering, pp: 1-6.

[9] Saeid Taheri, S. and Hosein Askarian-Abyaneh, 2011. Detecting of a Power Swing Phenomenon during Two Worst Simultaneous Faults Using System Characteristics, International Conference on Advancements in Electronics and Power Engineering (ICAEPE'2011) Bangkok Dec.

[10] Hadi Saadat, 1999. Power System Analysis, McGraw-Hill International Editions, Book. 


\section{Appendix A}

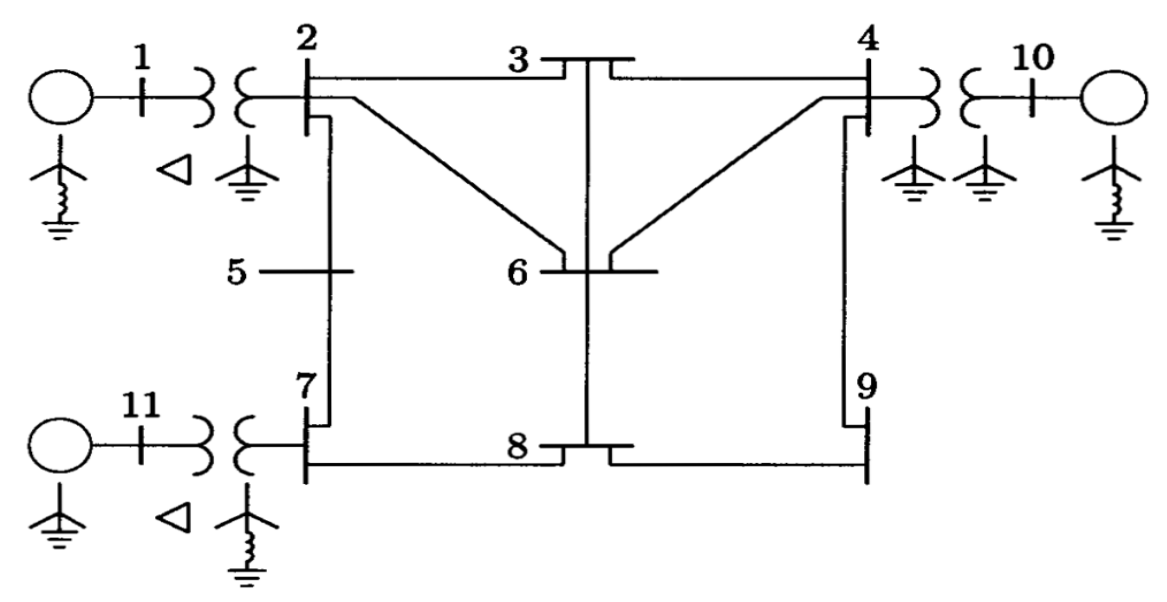

Fig. A: Single line diagram of 11-bus power system

Table A.1: Bus -Data for 11-line network

\begin{tabular}{|l|l|l|l|l|l|l|l|l|l|l|}
\hline Bus & Bus & Vl & Ang & Load & Gen & \multicolumn{2}{l|}{ Gen Mvar } & Injected \\
\hline No. & code & p.u. & Deg & MW & Mvar & MW & Mvar & Min & Max & Mvar \\
\hline 1 & 1 & 1.04 & 0 & 0 & 0 & 0 & 0 & 0 & 0 & 0 \\
\hline 2 & 0 & 1 & 0 & 0 & 0 & 0 & 0 & 0 & 0 & 0 \\
\hline 3 & 0 & 1 & 0 & 150 & 120 & 0 & 0 & 0 & 0 & 0 \\
\hline 4 & 0 & 1 & 0 & 0 & 0 & 0 & 0 & 0 & 0 & 0 \\
\hline 5 & 0 & 1 & 0 & 120 & 60 & 0 & 0 & 0 & 0 \\
\hline 6 & 0 & 1 & 0 & 140 & 90 & 0 & 0 & 0 & 0 & 0 \\
\hline 7 & 0 & 1 & 0 & 0 & 0 & 0 & 0 & 0 & 0 \\
\hline 8 & 0 & 1 & 0 & 110 & 90 & 0 & 0 & 0 & 0 \\
\hline 9 & 0 & 1 & 0 & 80 & 50 & 0 & 0 & 0 & 0 & 0 \\
\hline 10 & 2 & 1.035 & 0 & 0 & 0 & 200 & 0 & 0 & 180 & 0 \\
\hline 11 & 2 & 1.03 & 0 & 0 & 0 & 160 & 0 & 0 & 120 & 0 \\
\hline
\end{tabular}

Table A.2: Line -Data of the 11-Line network

\begin{tabular}{|c|c|c|c|c|c|c|}
\hline Bus no. & Bus no. & $\mathrm{R} 1$ & $\mathrm{X} 1$ & R0 & $\mathrm{X} 0$ & $\mathrm{~B} / 2$ \\
\hline 1 & 2 & 0 & 0.06 & 0 & 0.6 & 0 \\
\hline 2 & 3 & 0 & 0.3 & 0 & 0.6 & 0.0004 \\
\hline 2 & 5 & 0 & 0.15 & 0 & 0.3 & 0.0002 \\
\hline 2 & 6 & 0 & 0.45 & 0 & 0.9 & 0.0005 \\
\hline 3 & 4 & 0 & 0.4 & 0 & 0.8 & 0.0005 \\
\hline 3 & 6 & 0 & 0.4 & 0 & 0.8 & 0.0005 \\
\hline 4 & 6 & 0 & 0.6 & 0 & 1 & 0.0008 \\
\hline 4 & 9 & 0 & 0.7 & 0 & 1.1 & 0.0009 \\
\hline 4 & 10 & 0 & 0.08 & 0 & 0.08 & 0 \\
\hline 5 & 7 & 0 & 0.43 & 0 & 0.8 & 0.0003 \\
\hline 6 & 8 & 0 & 0.48 & 0 & 0.95 & 0 \\
\hline 7 & 8 & 0 & 0.35 & 0 & 0.7 & 0.0004 \\
\hline 7 & 11 & 0 & 0.1 & 0 & 0.1 & 0 \\
\hline 8 & 9 & 0 & 0.48 & 0 & 0.9 & 0 \\
\hline
\end{tabular}

Table A.3: G.T-Data for 11-line network

\begin{tabular}{|l|l|l|l|l|l|}
\hline $\begin{array}{l}\text { Bus } \\
\text { No. }\end{array}$ & $\begin{array}{l}\text { R0 } \\
\text { p.u. }\end{array}$ & $\begin{array}{l}\text { X0 } \\
\text { p.u. }\end{array}$ & $\begin{array}{l}\text { R1 } \\
\text { p.u. }\end{array}$ & $\begin{array}{l}\text { X1 } \\
\text { p.u. }\end{array}$ & $\begin{array}{l}\text { Xn } \\
\text { p.u. }\end{array}$ \\
\hline 1 & 0 & 0.2 & 0 & 0.06 & 0.05 \\
\hline 10 & 0 & 0.15 & 0 & 0.04 & 0.05 \\
\hline 11 & 0 & 0.25 & 0 & 0.08 & 0 \\
\hline
\end{tabular}

